\begin{tabular}{|c|c|}
\hline Title & Isolation and culture of microvascular endothelial cells from murine inguinal and epididymal adipose tissues \\
\hline Author(s) & $\begin{array}{l}\text { Kajimoto, Kazuaki; Hossen, Md. Nazir; Hida, Kyoko; Ohga, Noritaka; A kita, Hidetaka; Hyodo, Mamoru; Hida, } \\
\text { Y asuhiro; Harashima, Hideyoshi }\end{array}$ \\
\hline Citation & $\begin{array}{l}\text { Journal of Immunological Methods, 357(1-2), 43-50 } \\
\text { https://doi.org/10.1016/.jim.2010.03.011 }\end{array}$ \\
\hline Issue Date & 2010-05-31 \\
\hline Doc URL & http://hdl.handle.net/2115/43265 \\
\hline Type & article (author version) \\
\hline Additional Information & There are other files related to this item in HUSCAP. Check the above URL. \\
\hline File Information & JIM357-1-2_43-50.pdf \\
\hline
\end{tabular}

Instructions for use 
Journal of Immunological Methods

Technical Notes

\section{Isolation and Culture of Microvascular Endothelial Cells from Murine Inguinal and Epididymal Adipose Tissue}

Kazuaki Kajimoto ${ }^{\mathrm{a}}$ *, Md. Nazir Hossen ${ }^{\mathrm{a}}$, Kyoko Hida ${ }^{\mathrm{b}}$, Noritaka Ohga ${ }^{\mathrm{b}}$, Hidetaka Akitac, Mamoru Hyodo ${ }^{\text {a }}$ Yasuhiro Hida ${ }^{\mathrm{d}}$, Hideyoshi Harashima ${ }^{\mathrm{a}, \mathrm{c}}$

${ }^{\mathrm{a}}$ Laboratory of Innovative Nanomedicine, Faculty of Pharmaceutical Sciences, Hokkaido university, Kita 12, Nishi 6, Kita-ku, Sapporo, Hokkaido 060-0812, Japan

${ }^{\mathrm{b}}$ Division of Vascular Biology, Graduate School of Dental Medicine, Hokkaido university, Kita 13, Nishi 7, Kita-ku, Sapporo, Hokkaido 060-8586, Japan

${ }^{c}$ Laboratory for Molecular Design of Pharmaceutics, Faculty of Pharmaceutical Sciences, Hokkaido University, Kita 12, Nishi 6, Kita-ku, Sapporo, Hokkaido 060-0812, Japan

${ }^{\mathrm{d}}$ Department of Surgical Oncology, Graduate School of Medicine, Hokkaido University, Kita 15, Nishi 7, Kita-ku, Sapporo, Hokkaido 060-8638, Japan

*Correspondence:

Kazuaki Kajimoto, Ph.D.

Laboratory of Innovative Nanomedicine,

Faculty of Pharmaceutical Sciences, Hokkaido University

Kita 12, Nishi 6, Sapporo, Kita-ku, Hokkaido 060-0812, Japan

TEL: +81-11-706-3704

FAX: +81-11-706-3704

E-mail: k-kajimo@pharm.hokudai.ac.jp 


\begin{abstract}
Adipose tissue has long been considered to be a simple tissue that contains adipocytes. Because of this, the isolation and characterization of microvascular endothelical cells, which are also present in adipose tissue, has been neglected, even though they are components of a capillary network that surrounds each individual adipocyte. Here we report on a protocol for producing highly purified murine microvascular endothelial cells (MECs) from diverse sites of murine adipose tissues including inguinal and epididymal adipose tissue. The method is based on a combination of negative and positive immunomagnetic selection. The protocol involves the preparation of a single cell suspension (digestion, filtration and density gradient centrifugation), immunomagnetic enrichment of the $\mathrm{CD}^{4} 5^{-}$cell population and the purification of the MECs by a combination of common specific markers CD31, CD102 and isolectin B4. The isolated MECs can be successively cultured for 10 to 12 passages without any detectable changes in morphology and phenotype. Therefore, the method described herein represents a protocol for the isolation and long-term maintenance of highly pure mouse MECs in high yields from adipose tissues.
\end{abstract}

\title{
Keywords
}

Endothelial cell, Adipose tissue, Isolation, Primary culture 


\section{Introduction}

Adipose tissue is a unique organ, which is mainly composed of adipocytes supported by stromal vascular tissue, which contains vascular endothelial cells, macrophages, and stem cells (Crandall et al., 1997). Recent studies have proposed that the growth of adipose tissue is angiogenesis-dependent (Rupnick et al., 2002; Neels et al., 2004) and macrophage infiltration is closely related to angiogenesis and adipogenesis in the tip region of adipose tissue (Cho et al., 2007). Furthermore, adipose tissue is widely distributed in the body, and it can be generally classified as visceral and subcutaneous adipose tissue. It was recently reported that the gene expression profiles from these distinct fat depots were completely different (Gesta et al., 2006), indicating that adipose tissue is not a homogeneous organ and that the relationships between different fat depots and disease, such as diabetes or hyperlipidemia, can vary. Therefore, it is possible that, not only adipocytes and macrophages, but also microvascular endothelial cells (MECs) play a crucial role in the development or function of adipose tissue, and that the physiological function of MECs may also vary with individual fat depots. However, little is known concerning the nature of MECs in adipose tissue because they are present in low levels.

Techniques and strategies for the isolation of MECs from adipose tissue have been reported, in attempts to address this issue (Launder et al., 1987; Arts et al., 2001; Frye and Patrick, 2002). However, no reports are available that describe methods for isolating MECs from multiple parts of adipose tissue. In this report, we report on a method for accomplishing this, which is based on a combination of negative and positive immunomagnetic selection and permits the consistent isolation of pure MECs from murine inguinal and epididymal adipose tissue.

\section{Materials and Methods}

\subsection{Materials}

Collagenase (Type II) and Histopaque-1077 solution was obtained from SIGMA (St. Louis, MO, USA). Hanks' balanced salt solution (HBSS) was purchased from Wako Purechemicals 
(Osaka, Japan). Fluorescein isothiocyanate (FITC)-labeled isolectin B4 [Griffonia (Bandeiraea) simplicifolia lectin I] (GSI-B4) was purchased from Vector Laboratories (Burlingame, CA, USA). MidiMACS separation units, LD columns, FcR Blocking Reagent (mouse), goat anti-rat IgG Microbeads, and anti-FITC Microbeads were all obtained from Miltenyi Biotec (Auburn, CA, USA).

\subsection{Antibodies}

The following antibodies were purchased from Biolegend (San Diego, CA, USA): purified anti-CD31 (PECAM-1) (clone MEC13.3); purified or FITC-labeled anti-CD45 (LCA) (clone 30-F11); purified anti-CD102 (ICAM-2) (clone 3C4 (MIC2/4)); purified anti-CD105 (Endoglin) (clone MJ7/18); purified anti-CD106 (VCAM-1) (clone 429(MVCAM.A)); and purified anti-CD309 (VEGFR-2). The purified anti-CD34 (clone RAM34), anti-CD90 (Thy-1) (clone OX-7), and anti-CD144 (VE-cadherin) (clone BV13) were obtained from BD Pharmingen (San Jose, CA, USA), Millipore (Billerica, MA, USA), and eBioscience (San Diego, CA, USA), respectively.

\subsection{Animals}

Eight to nine week old male C57BL/6J mice were purchased from SLC Japan (Shizuoka, Japan). All animal procedures were carried out in accordance with the guidelines of the institutional animal care and research advisory committee of Hokkaido University, Sapporo, Japan.

\subsection{Isolation of stromal-vascular fraction (SVF) from murine inguinal and epididymal adipose tissue}

Twenty mice were used in the procedure. The mice were killed under anesthesia and adipose tissue excised from the inguinal and epididymal regions. These excised tissue samples were 
pooled in ice-cold HBSS, and then minced and digested with $20 \mathrm{ml}$ of $0.1 \%$ collagenase solution (pH 7.4) composed of $123 \mathrm{mM} \mathrm{NaCl}, 9.8 \mathrm{mM} \mathrm{KCl}, 1.3 \mathrm{mM} \mathrm{CaCl}, 5 \mathrm{mM}$ D-(+)-glucose, $100 \mathrm{mM}$ HEPES, and 2\% bovine serum albumin (BSA) for $30 \mathrm{~min}$ at $37^{\circ} \mathrm{C}$ using a water bath shaker. The tubes were agitated for a few seconds at 5 min intervals during this incubation. After digestion, undigested tissue was removed from the cell suspension by filtration through a $100 \mu \mathrm{m}$ cell strainer (BD Falcon, San Jose, CA, USA). The filtered cell suspension was then centrifuged for $10 \mathrm{~min}$ at 300x g. After removal of the floating cell layer and supernatant, the cell pellet was resuspended in $10 \mathrm{ml}$ of HBSS, and then filtered through a $40 \mu \mathrm{m}$ cell strainer (BD Falcon). The filtered cell suspension was layered on top of a Histopaque-1077 solution (10 ml) in a $50 \mathrm{ml}$ tube, and then centrifuged at 400x g for $30 \mathrm{~min}$ at room temperature. The SVF cell layer at the gradient interface was collected and washed with HBSS. Cell counts and viability assessment were performed.

\subsection{Negative selection for enrichment of $\mathrm{CD}^{-} 5^{-}$cells containing MECs}

The collected SVF was centrifuged at 300x g for $10 \mathrm{~min}$ and the cell pellet resuspended in MACS buffer (0.5\% BSA and 2 mM EDTA in PBS, pH7.2) $\left(10^{7}\right.$ cells / $\left.100 \mu \mathrm{l}\right)$, and then incubated with FcR blocking reagent for $10 \mathrm{~min}$ at $4^{\circ} \mathrm{C}$ according to the manufacturer's recommended protocol. The blocked cells were incubated with FITC-labeled rat anti-mouse CD45 antibody at a 1:10 dilution for $30 \mathrm{~min}$ at $4^{\circ} \mathrm{C}$. After washing, the cells were incubated with anti-FITC Microbeads for 15 min at $4^{\circ} \mathrm{C}$. During this incubation, the LD columns were loaded onto MidiMACS separation units and each column was washed with $2 \mathrm{ml}$ of MACS buffer. After the beads had reacted, the cells were washed with $1 \mathrm{ml}$ of MACS buffer and the cell pellet was resuspended in $500 \mu 1$ of buffer. The resulting suspension was then applied to the washed column. Since the Microbead-coated $\mathrm{CD}_{4} 5^{+}$cells were retained on the LD columns, the flow-through (CD45 cell fraction) was collected in new tube. The columns were washed twice with $1 \mathrm{ml}$ portions of MACS buffer and the total effluent was also collected. 
Flow cytometry was used to confirm the separation.

\subsection{Positive selection for the purification of MECs}

The CD45 ${ }^{-}$cells were washed, resuspended in MACS buffer $\left(10^{7}\right.$ cells / $\left.100 \mu 1\right)$, and then incubated with rat anti-mouse CD31, rat anti-mouse CD102 antibody and FITC-labeled isolectin B4 (GSI-B4) for $30 \mathrm{~min}$ at $4^{\circ} \mathrm{C}$. Each antibody and GSI-B4 was used at a 1:10 dilution and 20 $\mu \mathrm{g} / \mathrm{ml}$, respectively. After washing, the cells were incubated with goat anti-rat IgG Microbeads and anti-FITC Microbeads for $15 \mathrm{~min}$ at $4^{\circ} \mathrm{C}$. After the beads reacted, the cells were washed and applied to the equilibrated LD column. Since the Microbead-coated CD31 ${ }^{+}, \mathrm{CD}_{102}{ }^{+}$, and/or GSI-B4-binding cells were retained on the LD columns, the flow-through was discarded. The columns were washed twice with $1 \mathrm{ml}$ portions of MACS buffer, and the magnetically labeled cells were then flushed out by firmly applying the plunger supplied with the columns. Flow cytometry was used to confirm the separation.

\subsection{Primary culture of freshly isolated MECs}

Immediately after separation, the collected cells were washed and resuspended in EGM-2MV media (Lonza, Walkersville, MD, USA) supplemented with 10\% fetal bovine serum (GIBCO, Grand Island, NY, USA) and $0.1 \mathrm{mg} / \mathrm{ml}$ kanamicin sulfate (Wako Purechemicals). The cells were seeded onto $60 \mathrm{~mm}$ culture dishes coated with $1.5 \%$ gelatin (SIGMA) and $10 \mu \mathrm{g} / \mathrm{ml}$ of human fibronectin (Asahi Glass, Tokyo, Japan). Nonadherent cells were removed on day 2 by washing with PBS. On day 4 or 5, the cells were trypsinized and passaged to $100 \mathrm{~mm}$ culture dishes coated with the above described reagents. The cells were subsequently passaged at a 1:3 ratio until they reached $80-90 \%$ confluence. Cells at 6 - 10 passage numbers were collected by brief trypsinization and analyzed by flow cytometry, as described below.

\subsection{Flow cytometry}


To confirm the efficiency of the negative selection, the fractions before and after magnetic cell sorting were analyzed using a FACS Calibur flow cytometer (BD Biosciences, San Jose, CA, USA).

To determine which surface markers can be adapted to perform a positive selection, freshly isolated $\mathrm{CD}^{-} 5^{-}$cells were examined for surface marker expression using flow cytometry. $\mathrm{CD} 45^{-}$cells were incubated with the following primary antibodies for $30 \mathrm{~min}$ at $4{ }^{\circ} \mathrm{C}$ : rat anti-mouse CD31; rat anti-mouse CD102; or rat anti-mouse CD105 at 1:10 dilution. After washing, the cells were incubated with Alexa488-labeled goat anti-rat IgG (Invitogen, Grand Island, NY, USA) for a further $30 \mathrm{~min}$ at $4^{\circ} \mathrm{C}$. In addition, the $\mathrm{CD} 45^{-}$cells were also incubated with FITC-labeled GSI-B4 at a concentration of $20 \mu \mathrm{g} / \mathrm{ml}$ for $30 \mathrm{~min}$ at $4^{\circ} \mathrm{C}$. After washing, the cells were analyzed using a FACS Calibur.

To determine the efficiency of the positive selection, the fractions before and after magnetic cell sorting were stained by FITC-conjugated GSI-B4 and analyzed using a FACS Calibur.

To examine the nature of the cultured MECs, the cells at 6 - 10 passage numbers were collected by brief trypsinization. After washing, the cells were incubated with FcR blocking reagent for $10 \mathrm{~min}$ at $4^{\circ} \mathrm{C}$ and then incubated with pair of primary and secondary antibodies or FITC-labeled GSI-B4, as described above. After washing, the cells were analyzed using a FACS Calibur.

\subsection{Immunofluorescent staining}

Immunohistochemical analyses were performed using a confocal microscopy-based method for the three-dimensional visualization of fixed adipose tissue as described previously (Nishimura et al., 2007). Briefly, C57BL/6J mice were killed under anesthesia and adipose tissue excised from the inguinal and epididymal regions. These excised tissue samples were washed with HBSS and minced into small pieces $(2-3 \mathrm{~mm})$ using a scalpel. The isolated tissue pieces were fixed in 3\% paraformaldehyde in PBS for 45 min at room temperature. 
After washing with PBS three times, the fixed pieces were exposed to 5\% glycine in PBS for 30 min to quench any free aldehyde groups. After washing, the tissue pieces were permeabilized with $1 \%$ Triton X-100 in PBS for 10 min, and blocked by treatment with $1 \%$ bovine serum albumin (BSA) and 0.3\% Triton X-100 in PBS for $1 \mathrm{hr}$ at room temperature. After blocking, the tissue pieces were incubated with primary antibodies for CD31, CD34, CD45, CD90, CD102, CD105, CD106, CD144 or CD309 in blocking solution (1:100 dilution) for $12 \mathrm{hr}$ at $4^{\circ} \mathrm{C}$. The tissue pieces were washed with PBS three times and incubated with Alexa 568-labeled secondary antibody (Invitrogen) in blocking solution (1:100 dilution) for $1 \mathrm{hr}$ at room temperature. The specimens were washed with PBS three times and then stained with 20 $\mu \mathrm{g} / \mathrm{ml}$ of Alexa 647-conjugated GSI-B4 (Invitrogen) for $1 \mathrm{hr}$ at room temperature. For lipid staining, the specimens were placed in $5 \mu \mathrm{M}$ of BODIPY493/503 (Invitrogen) for $1 \mathrm{hr}$, and nuclei were then counterstained with $40 \mu \mathrm{M}$ of Hoechst 33342 (Nacali Tesque) for 1 hr. After washing, the specimens were placed on micro cover grasses (Matsunami) and observed by confocal laser scan microscopy (model A1, Nikon).

\subsection{Reverse transcription and polymerase chain reaction (RT-PCR)}

Total RNA samples were prepared from cultured MECs at passage numbers 6 - 10 using the TRIzol reagent (Invitrogen), according to the manufacturer's protocol. To eliminate genomic DNA, $10 \mu \mathrm{g}$ of total RNA was treated with Turbo DNase (Ambion) for $30 \mathrm{~min}$ at $37^{\circ} \mathrm{C}$. RNA integrity and quality were assessed by electrophoresis, and $1 \mu \mathrm{g}$ of the DNase-treated RNA was then reverse transcribed into cDNA using a High Capacity RNA-to-cDNA Kit (Applied Biosystems). The following gene-specific primers were used: CD34-F: 5'-gggagccaccagagctattc, CD34-R: 5'-caccacatgttgtcttgctga; CD90-F: 5'-atccagcatgagttcagcct, CD90-R: 5'-caaccagtcacagagaaatgaagt; CD105-F: 5'-ctctgaggtcacagtccagc, CD105-R: 5'-agaatagatgtaaccagagtgcagc; $\quad$ CD144-F: 5'-cgcaccaggtattcaagcgatctg, $\quad$ CD144-R: 5'-cacagtggctgtgcctgactct; CD146-F： 5'- ggctaccccattcctcaagt, CD146-R: 5'- 
gttgatagtgaagtggggttgag. PCR products were subjected to 2\% agarose gel electrophoresis.

\section{Results}

\subsection{Negative selection for enrichment of $\mathrm{CD}^{-} 5^{-}$cells including microvascular endothelial} cells

The stromal-vascular fraction (SVF) obtained from inguinal and epididymal adipose tissue by collagenase digestion and single sucrose step-gradient centrifugation with Histopaque-1077 contains $\mathrm{CD}_{4} 5^{+}$hematopoietic cells. Based on the FACS analysis before negative selection, the positive ratio of CD45 in SVF from inguinal and epididymal adipose tissue were determined to be $31.8 \%$ and $32.9 \%$, respectively (Fig. 1). The positive ratio in the flow through fraction after MACS separation from the two types of adipose tissue was decreased to $4.0 \%$ (inguinal) and 1.5\% (epididymal) (Fig. 1), indicating that $\mathrm{CD}^{-} 5^{-}$cells including MECs were enriched by about $1.4-1.5$ fold as the result of this negative selection step.

\subsection{Characterization of $\mathrm{CD}^{-}$cell populations from inguinal and epididymal adipose} tissue for the positive selection of MECs

$\mathrm{CD}^{-} 5^{-}$cell populations from adipose tissue include not only MECs but other cell populations, such as pericytes and stem cells as well. Therefore, purifying the $\mathrm{CD} 45^{-}$cell fraction is critical for obtaining a pure MEC population. However, information concerning specific maker molecules that are expressed in both MECs from inguinal and epididymal adipose tissue is not available. Therefore, we attempted to identify common markers that could be useful in the positive selection of MECs from inguinal and epididymal adipose tissues.

We first examined the expression of some markers in two types of adipose tissues by immunohistological analysis. The findings indicated that CD31 (Supplementary Fig. 1) and CD102 (Fig. 2A) were specifically detected in microvessels and colocalized with the area stained by GSI-B4 in both adipose tissues, although CD34, CD45 and CD90 were detected in, 
not only microvessels, but in the other stromal area (Supplementary Fig. 1). Moreover, CD105, CD106, CD144 and CD309 were not detected to a significant extent (Supplementary Fig. 1). These results suggest that CD31, CD102 and GSI-B4 are suitable for the purification of MECs from SVF.

Although CD105 was not detected clearly in microvessels by histological analysis, it was previously reported that CD105 was also expressed in both of CD31 positive and negative cells from adipose tissue (Boquest et al., 2005). Therefore, we next analyzed the patterns of these four markers CD31, CD102, CD105 and GSI-B4 in CD45 cell populations from inguinal and epididymal adipose tissue using flow cytometry (Fig. 2B). Among these, we identified three markers, CD31, CD102 and GSI-B4, the patterns of which were similar with each other. Among the markers analyzed, only CD105 showed a different expression pattern from the other markers. These results suggest that the positive cell populations of CD31, CD102 and GSI-B4 were very similar, but that the $\mathrm{CD} 105^{+}$cell population was different from the other three markers. Therefore, we selected these three markers (CD31, CD102 and GS-IB4) for use in the positive selection of MECs.

\subsection{Characterization of isolated cells from inguinal and epididymal adipose tissue immediately after MACS separetion}

To purify MECs from the $\mathrm{CD}^{-} 5^{-}$cell fraction, we used the above three markers for positive selection. We analyzed and compared the ratio of GSI-B4-positive cells between before and after MACS separation using flow cytometry (Fig. 3A). The positive ratios of GSI-B4 in the CD45 cell fraction from inguinal and epididymal adipose tissue were determined to be $27.2 \%$ and $21.4 \%$, respectively. In contrast, the positive ratio in the trapped fraction after MACS separation from the two types of adipose tissue was increased to $70.7 \%$ (inguinal) and $74.2 \%$ (epididymal), indicating that MECs were enriched by about 2.6 - 3.5 fold as the result of this

positive selection step. In addition, the positive cell ratio in the flow through fraction not 
trapped by MACS columns was 12.1\% (inguinal) and 7.5\% (epididymal) (Supplementary Fig. 2). These results indicate that approximately $90 \%$ of the MECs contained in the $\mathrm{CD}^{-} 5^{-}$cell fraction was separated and collected by this step. The viability of the isolated cells immediately after positive selection was determined to be $83.0 \%$ (inguinal) and $84.1 \%$ (epididymal) (Supplementary Table 1).

\subsection{Morphological characteristics of isolated MECs from inguinal and epididymal adipose} tissue

After positive selection by magnetic cell sorting using combination of above three markers, isolated cells were propagated for $4-5$ days. As shown in Fig. 3B, our isolated cells showed highly homogeneous morphologies. The cells were passaged up to ten times when $80-90 \%$ confluent, resulting in pure culture at each passage and after several passages cells retained its characteristics.

\subsection{Molecular characteristics of cultured MECs from inguinal and epididymal adipose} tissue

The purity of the endothelial cells isolated from inguinal and epididymal adipose tissue was determined by flow cytometry on the basis of CD31, CD102 and GSI-B4, since our immunomagnetic selection was established using these molecules. As shown in Fig. 3C, both of the endothelial cell cultures (passages 6 - 10) were positive for all three markers, as evidenced by flow cytometry. Several previously reported adipose endothelial cell markers were analyzed by RT-PCR (Fig. 3D). As a result, all five markers were expressed in both populations of MECs. These results strongly suggest that our MECs that were isolated from two types of adipose tissue were highly pure and that their endothelial characteristics were retained up to passages $6-10$. 


\section{Discussion}

The goal of the present study was to establish a reliable protocol for the isolation and culturing of MECs from diverse sites of murine adipose tissues, including inguinal and epididymal adipose tissue. The strategy involves the preparation of a suspension of single cells (digestion, filtration and density gradient centrifugation), immunomagnetic enrichment of the $\mathrm{CD}^{-} 5^{-}$cell population and purification of the MECs by a combination of common specific markers CD31, CD102 antibody and GSI-B4.

It was previously reported that the use of three markers for the positive selection of MECs increases the efficiency and specificity of the isolation procedure as more magnets labeled on a cell surface would enhance the trapping efficiency (Marelli-Berg et al., 2000). In this previous report, the investigators isolated capillary ECs from murine lung and heart using a combination of an anti-CD31 antibody, anti-CD105 antibody and biotinylated GSI-B4 by magnetic bead separation. In their protocol, an overnight culture was employed to remove nonadherent cells, such as $\mathrm{CD}_{4} 5^{+}$hematopoietic cells, from the SVF. However, to collect adherent cells, tripsinazation is necessary for positive selection. In addition, in this method, macrophages, which are $\mathrm{CD} 45^{+}$adherent cells, cannot be removed. For the isolation of pure MECs, it is important that the MECs population is enriched as much as possible prior to positive selection, since little information is available concerning highly specific endothelial cell markers. Therefore, we performed CD45 negative selection of SVF before positive selection and, as a result, it was possible to collect more than $95 \%$ of the $\mathrm{CD} 45^{-}$cell population (Fig.1) and permit the working time to be shortened substantially. For positive selection, these investigators used CD105 from lung and heart as an isolation marker. In this study, we histologically analyzed the expression of 10 types of markers in inguinal and epididymal adipose tissues (Fig. 2A and Supplementary Fig. 1) and confirmed that CD31 and CD102 were specifically detected in the microvessels as well as GSI-B4, as evidenced by the immunofluorescent staining of inguinal and epididymal adipose tissues. In this analysis, CD105 was not clearly detected in both types 
of adipose tissues. However, it was previously reported that CD105 is expressed in both of CD31 positive and negative cell fractions from adipose tissue (Boquest et al., 2005), hence, we next examined the characteristics of the $\mathrm{CD} 45^{-}$cell population by flow cytometry (Fig. 2B). The pattern for CD105 was different among several markers in both cases of inguinal and epididymal cells. On the other hand, the patterns for CD31, CD102 and GSI-B4 were extremely similar with each other. These findings are consistent with the previous report that CD31- stromal cells included in the $\mathrm{CD}^{-} 5^{-}$cell fraction from human adipose tissue also expressed CD105 (Boquest et al., 2005). The reason for why CD105 was not histologically detected in both adipose tissues is unclear at present. However, it is noteworthy in this regard that no reports have appeared to show that CD102 is expressed in microvascular endothelial cells in both inguinal and epididymal adipose tissue and applicable to the isolation of MECs from these adipose tissues.

Based on these results, we used three markers (CD31, CD102 and GSI-B4) for the positive selection of MECs from the $\mathrm{CD}^{4} 5^{-}$cell fraction. As a result, about $90 \%$ of the MECs (GSI-B4-positive cells) contained in the CD45- cell fraction were trapped by MACS columns, and $>70 \%$ of the separated cells were confirmed to be GSI-B4-positive by flow cytometry (Fig. 3A). Furthermore, we examined the advantages of combining the three selection markers. As shown in Supplementary Fig. 2 and Supplementary Table 1, the positive cell ratio of GSI-B4 in the isolated cells just after MACS separation was similar (70 - 75\%) between the combined application and the use of each single marker. The GSI-B4-positive cell ratio in the flow through fraction was also similar (around 10\%) in all cases. These results indicate that there is certain limit in the improvement of purification efficiency and recovery rate by the combined use of several markers. However, unexpectedly, the viability of the isolated cells just after MACS separation was greatly improved in case of the combined application (Supplementary Table 1). No reports have appeared to show similar results to this finding, and further investigations will be required to elucidate the protective effect. 
The cultured MECs from inguinal and epididymal adipose tissue showed a low expression of CD31 (Fig. 3C). After several passages with tripsinization, the expression decreased several times due to be damage incurred by enzymatic digestion, particularly tripsin, as described in a previous report (Marelli-Berg et al., 2000). It is well known that the magnetic sorting technique is not capable of completely purifying only one cell population from a heterogeneous cell mixture because of non-specific antibody binding or undesired cell-cell interactions. However, the endothelial cell characteristics in $>95 \%$ of our isolated and cultured MECs were evident by FACS analysis (Fig. 3C), indicating that the level is extremely low, even if other cell populations are present with the isolated MECs as contaminants. The positive cell ratio of GSI-B4 just after positive selection was $70-75 \%$ (Fig. 3A), indicating that the positive cell ratio was increased during culture and propagation. However, these results appear to be reasonable, since about $20 \%$ of the collected cells were already dead just after MACS separation (Supplementary Table 1).

In conclusion, our isolation and culture methods described herein can be used to isolate highly pure MECs from diverse sites of murine adipose tissues including inguinal and epididymal adipose tissue. A reliable method for isolating MECs will allow investigators to elucidate the physiological and pathophysiological role of MECs in the development of adipose tissue and the relationship between these and various diseases.

\section{Acknowledgments}

This study was supported by grants from the Special Education and Research Expenses of the Ministry of Education, Culture, Sports, Science and Technology of Japan. We also thank Dr. Milton Feather for editing this manuscript. We also thank him for his kind advice in preparing this manuscript. 


\section{References}

Arts, C.H.P., Heijnen-Snyder, G.J., Joosten, P.P.A.H., Verhagen, H.J.M., Eikelboom, B.C., Sixma, J.J. and de Groot, P.G. (2001) A novel method for isolating pure microvascular endothelial cells from subcutaneous fat tissue ideal for direct cell seeding. Laboratory Investigation 81, 1461-1465.

Boquest, A.C., Shahdadfar, A., Fronsdal, K., Sigurjonsson, O., Tunheim, S.H., Collas, P. and Brinchmann, J.E. (2005) Isolation and transcription profiling of purified uncultured human stromal stem cells: Alteration of gene expression after in vitro cell culture. Molecular Biology of the Cell 16, 1131-1141.

Cho, C.H., Koh, Y.J., Han, J., Sung, H.K., Lee, H.J., Morisada, T., Schwendener, R.A., Brekken, R.A., Kang, G., Oike, Y., Choi, T.S., Suda, T., Yoo, O.J. and Koh, G.Y. (2007) Angiogenic role of LYVE-1-positive macrophages in adipose tissue. Circulation Research 100, E47-E57.

Crandall, D.L., Hausman, G.J. and Kral, J.G. (1997) A review of the microcirculation of adipose tissue: Anatomic, metabolic and angiogenic perspectives. Microcirculation 4, 211-232.

Frye, C.A. and Patrick, C.W. (2002) Isolation and culture of rat microvascular endothelial cells. In Vitro Cellular \& Developmental Biology-Animal 38, 208-212.

Gesta, S., Bluher, M., Yamamoto, Y., Norris, A.W., Berndt, J., Kralisch, S., Boucher, J., Lewis, C. and Kahn, C.R. (2006) Evidence for a role of developmental genes in the origin of obesity and body fat distribution. Proceedings of the National Academy of Sciences of the United States of America 103, 6676-6681.

Launder, T.M., Gegen, N.W., Knedler, A. and Harbeck, R.J. (1987) The Isolation and Characterization of Enriched Microvascular Endothelial-Cells from Mouse Adipose-Tissue - the Induction of Class-Ii Molecules of the Major Histocompatibility Complex (Mhc) by Interferon-Gamma (Ifn-Gamma). Journal of Immunological 
Methods 102, 45-52.

Marelli-Berg, F.M., Peek, E., Lidington, E.A., Stauss, H.J. and Lechler, R.I. (2000) Isolation of endothelial cells from murine tissue. Journal of Immunological Methods 244, 205-215.

Neels, J.G., Thinnes, T. and Loskutoff, D.J. (2004) Angiogenesis in an in vivo model of adipose tissue development. Faseb Journal 18, 983-+.

Nishimura, S., Manabe, I., Nagasaki, M., Hosoya, Y., Yamashita, H., Fujita, H., Ohsugi, M., Tobe, K., Kadowaki, T., Nagai, R. and Sugiura, S. (2007) Adipogenesis in obesity requires close interplay between differentiating adipocytes, strontal cells, and blood vessels. Diabetes 56, 1517-1526.

Rupnick, M.A., Panigrahy, D., Zhang, C.Y., Dallabrida, S.M., Lowell, B.B., Langer, R. and Folkman, M.J. (2002) Adipose tissue mass can be regulated through the vasculature. Proceedings of the National Academy of Sciences of the United States of America 99, 10730-10735. 


\section{Figure Legends}

Figure 1. Phenotypic characterization of the stromal-vascular fraction from murine inguinal and epididymal adipose tissue.

Single cell suspension and flow through fraction of CD45 negative selection from inguinal and epididymal adipose tissue were analyzed by flow cytometry.

Figure 2. Immunohistochemical and flow cytometric analysis of endothelial cell marker expression in murine inguinal and epididymal adipose tissue.

A) Paraformaldehyde-fixed inguinal or epididymal adipose tissues from C57B/6J mice were stained with antibodies against CD102 (green, Alexa568-labeled secondary antibody). The microvessels were stained by Alexa647-labeled GSI-B4 (red), the lipid was counterstained with BODIPY493/503 (blue), and the nuclei with Hoechst33342 (cyan). Scale bars represent 100 $\mu \mathrm{m}$. B) The $\mathrm{CD} 45^{-}$cells isolated from inguinal and epididymal adipose tissue were stained with antibodies specific for several markers.

Figure 3. Phenotypic and morphological characterization of the isolated and cultured MECs from murine inguinal and epididymal adipose tissue.

A) Immediately before and after positive selection, the cells from murine inguinal and epididymal adipose tissue were stained with FITC-conjugated GSI-B4 and analyzed by flow cytometry. B) Phase contrast microscopy images of isolated and cultured MECs from from murine inguinal (day 3) and epididymal (day 4) adipose tissue. The magnification of photomicrographs is $\mathrm{x} 40$. C) Cultured MECs from murine inguinal and epididymal adipose tissue (passages 6 -10) were stained with antibodies specific for several endothelial cell markers. D) Expression of several endothelial cell markers in the cultured MECs from murine inguinal and epididymal adipose tissue (passages 6 -10) were analyzed by RT-PCR. 


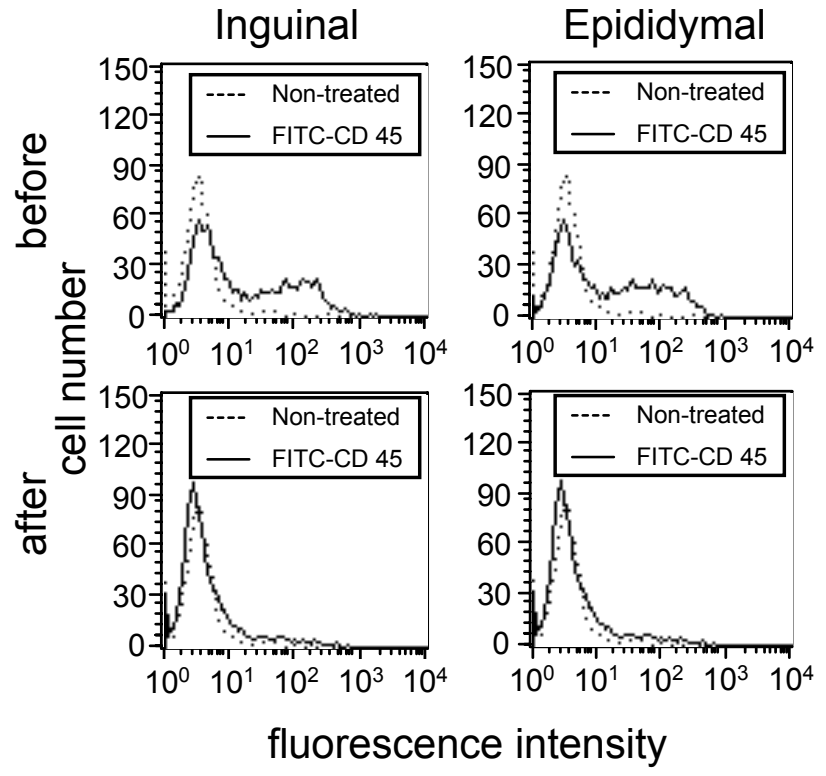

Fig. 1 
A

CD102

GSI-B4

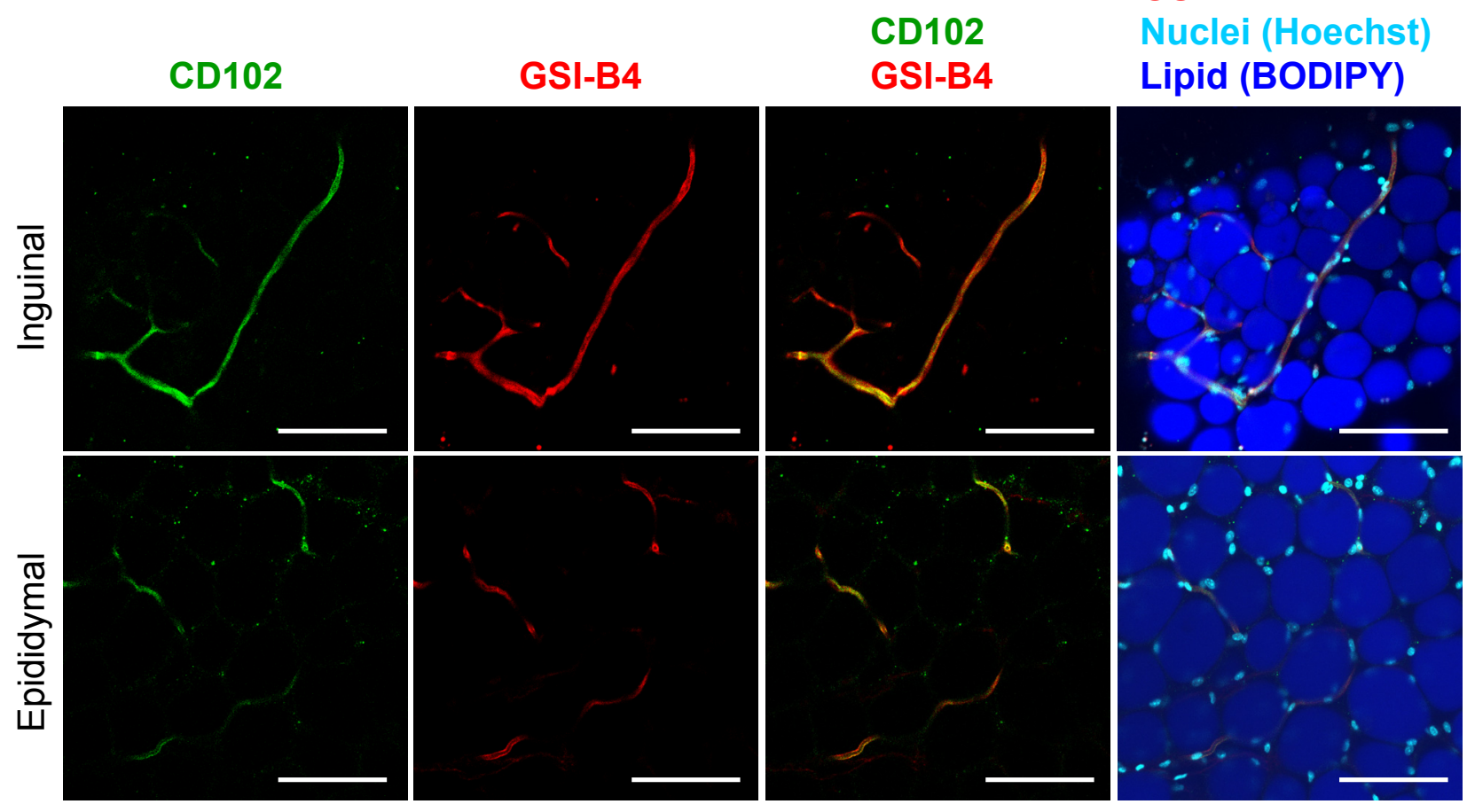

B

Inguinal
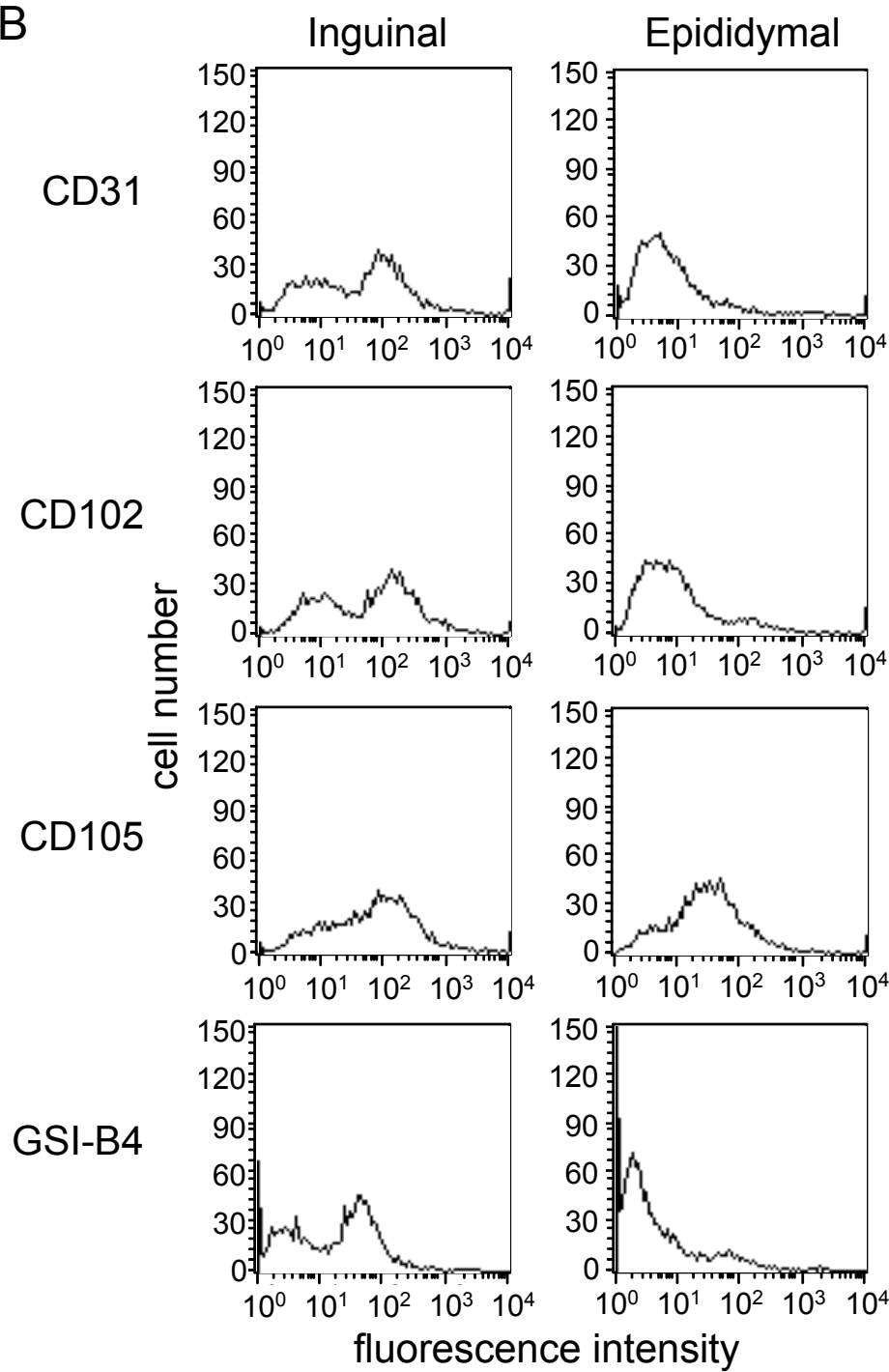

Fig. 2 
A

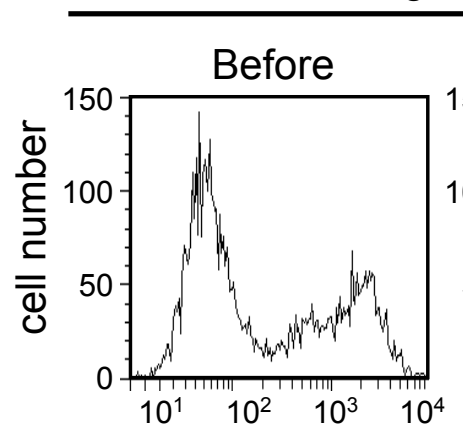

Inguinal

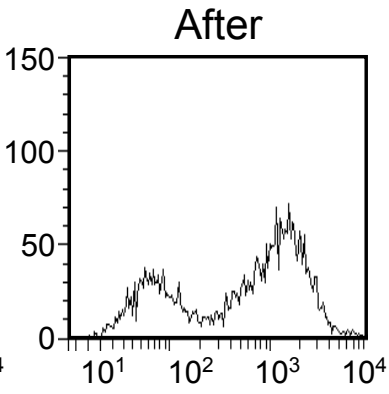

fluorescence intensity
Epididymal

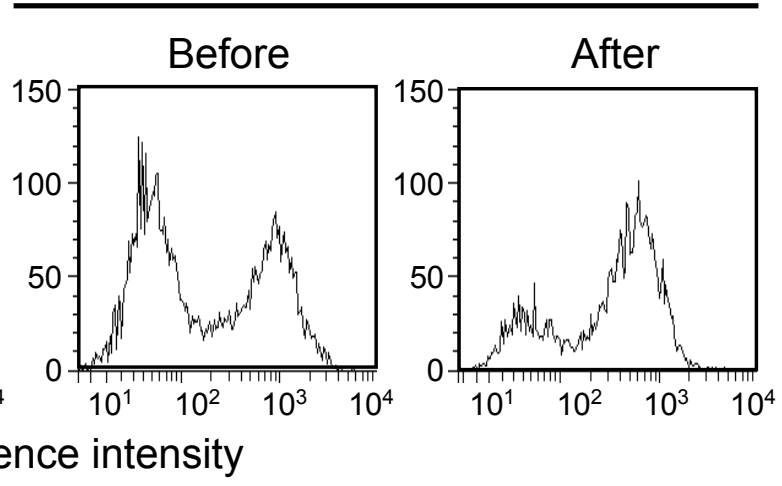

B

Inguinal

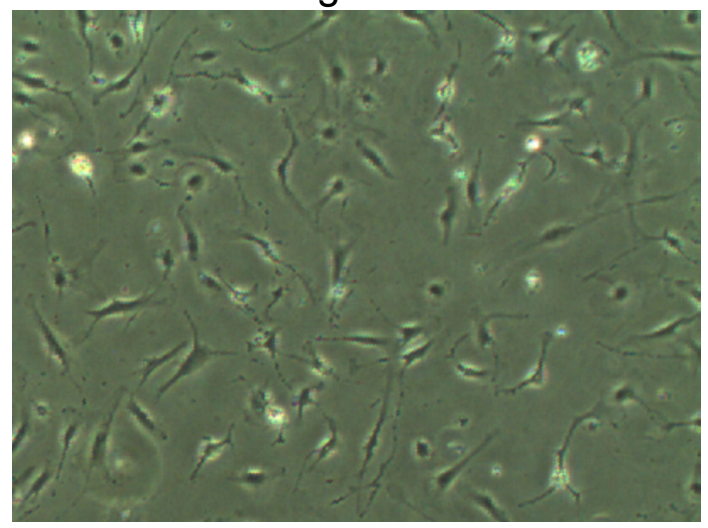

Epididymal

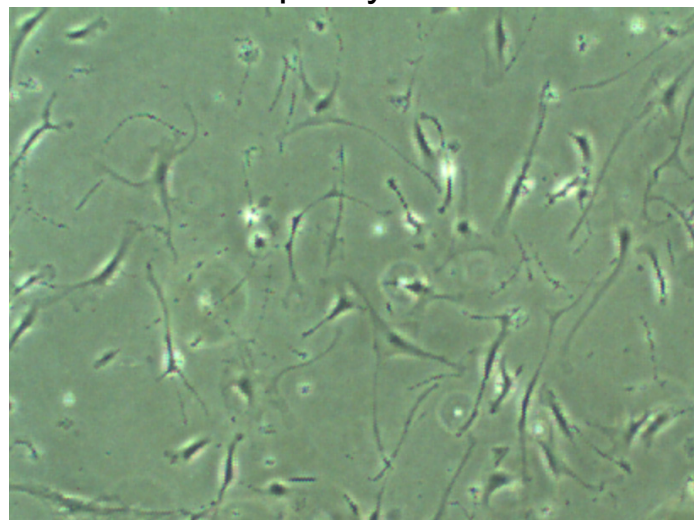

C
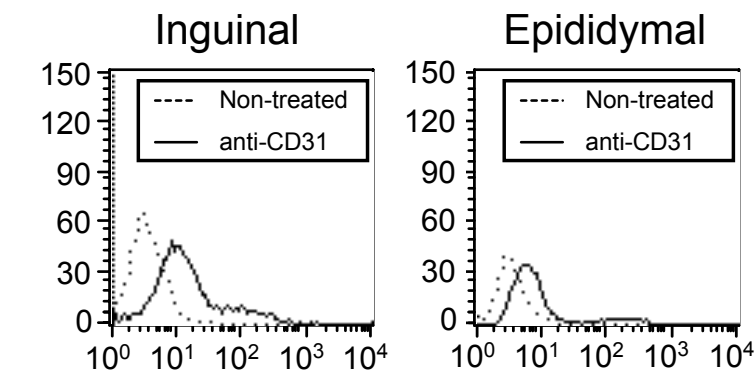

CD102
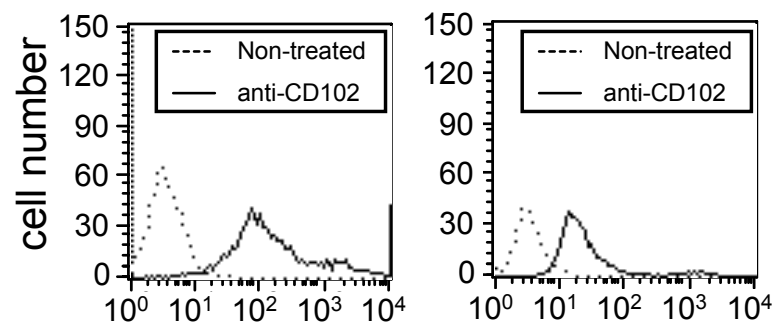

(1)
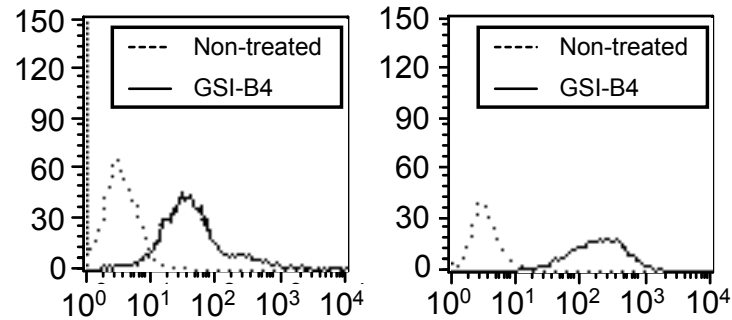

D

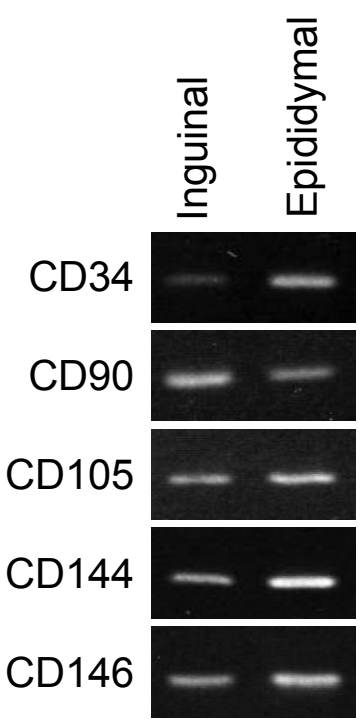

GSI-B4

fluorescence intensity

Fig. 3 


\section{Supplementary Figure legends}

\section{Supplementary Figure 1. Immunohistochemical analysis of CD markers in murine adipose}

tissue.

Paraformaldehyde-fixed (A) inguinal or (B) epididymal adipose tissues from C57B/6J mice were stained with antibodies against CD31, CD34, CD45, CD90, CD105, CD106, CD144 or CD309 (green, Alexa568-labeled secondary antibody). The microvessels were stained by Alexa647-labeled GSI-B4 (red). And, the lipid was counterstained with BODIPY493/503 (blue), and the nuclei with Hoechst33342 (cyan). Scale bars represent $100 \mu \mathrm{m}$.

\section{Supplementary Figure 2. Flow cytometric analysis of the obtained cell fraction immediately before and after positive selection.}

(A) After isolation of $\mathrm{CD} 45^{-}$cell fraction from murine inguinal and epididymal adipose tissue, the cells were stained with FITC-conjugated GSI-B4 and then analyzed by flow cytometry. The non-stained cells were also analyzed as a negative control. (B) After positive selection, the trapped and flow through fractions were stained with FITC-conjugated GSI-B4 and then analyzed by flow cytometry. We analyzed and compared between the combined application of 3 markers including CD31, CD102 and GSI-B4 or each single marker usage. 